\title{
Percutaneous and Surgical Tracheostomy in Elderly
}

\author{
Ali Bestemi Kepekci ${ }^{1,2 *}$ and Ahmet Hamdi Kepekci ${ }^{3,4}$ \\ ${ }^{1}$ Department of Anesthesia, Istanbul Yeni Yuzyil University, Turkey \\ ${ }^{2}$ Department of Anesthesiology and Reanimation, Meltem Hospital, Turkey \\ ${ }^{3}$ Department of Audiometry, Istanbul Yeni Yuzyil University, Turkey \\ ${ }^{4}$ Department of Otolaryngology, Meltem Hospital, Turkey
}

Submission: July 04, 2020; Published: July 22, 2020

*Corresponding author: Ali Bestemi Kepekci, Department of Anesthesiology and Reanimation \& Department of Anesthesia, Vocational Health High School, Istanbul Yeni Yuzyil University, Turkey

\section{Abstract}

Introduction: Tracheostomy is one of the most common procedures in geriatric patients receiving long-term mechanical ventilation. Percutaneous tracheostomy (PT) is increasingly used as an alternative to traditional surgical tracheostomy (ST). This study evaluated the complications, operative time, and functional outcomes of percutaneous and open surgical tracheostomy in elderly patients treated at intensive care units (ICUs).

Methods: The medical records of geriatric patients who underwent tracheostomy at ICUs were retrospectively reviewed. Patients included in the study were categorised into two groups: PT or ST. Patients' age, sex, APACHE II scores, number of intubated days, length of stay in ICU, mortality, operative times, and complications during and after the procedure were evaluated.

Results: A total of 47 patients were included in the study; the PT group comprised 27 patients, and the ST group comprised 20 patients. Overall, $55 \%$ of the patients died during the first 30 days after tracheostomy. The mean \pm SD operation time was $23.4 \pm 3.2 \mathrm{~min}$ for ST and $11.2 \pm$ $2.8 \mathrm{~min}$ for PT $(\mathrm{p}=0.00)$. No significant differences were observed in complication rates between the groups $(\mathrm{p}>0.05)$.

Discussion and Conclusion: There were no significant differences between the two methods in terms of complications. Nevertheless, PT had a shorter duration than ST. Among geriatric patients, tracheostomy does not increase survival and quality of life. In cases of prolonged intubation, tracheostomy should be planned considering the clinical benefits and risks for the patients.

Keywords: Geriatrics; Intubations; Intensive care units; Tracheostomy; Postoperative Complications

\section{Introduction}

Tracheostomy, one of the oldest surgical procedures, has long been used as routine treatment for elderly patients with severe respiratory failure [1]. Open surgery can be performed by surgeons in the form of surgical tracheostomy (ST). In the recent years, percutaneous tracheostomy (PT) has been performed by anesthesia and reanimation specialists at intensive care units (ICUs). The reported advantages of PT include a smaller skin incision as well as reduced tissue trauma, wound infection, and bleeding. An additional advantage of this method is that it can be applied at the bedside, which eliminates the risks associated with the transfer of patients while they are connected to a mechanical ventilator and thus reducing the need for personnel and saving time [1].
Although PT has become increasingly popular in the recent years, it is essential to appropriately select patients to undergo this procedure. While ST can be applied to every patient, PT cannot be applied to every patient. Anatomical variations, previous surgical procedures applied to the neck area, or presence of coagulopathy may not allow PT administration [2]. PT may need to be converted to ST because of complications such as bleeding, misplacement, hypotension, air embolism, apnoea, cardiac arrest, thyroid and cricoid cartilage damage, recurrent laryngeal nerve damage and posterior tracheal wall damage. The most common postoperative complications associated with tracheostomy are bleeding, subcutaneous emphysema, infection, pneumothorax, tracheal stenosis, and tracheoesophageal fistula [3-8]. 
In a study of 1236 patients, the intensive care general mortality rate was reported as $32 \%$ and the mortality rate of the geriatric group was reported as 59\% [9]. In another study involving 2098 patients, the mortality risk of elderly patients was 2.002 times higher than other patients. In another study involving 2098 patients, the mortality risk of elderly patients was 2.002 times higher than other patients. This risk has been attributed to the frequency of chronic diseases such as cardiovascular diseases, COPD, cerebrovascular diseases in patients 65 and older [10]. There are studies indicating that long-term intubation in geriatric patients should not the only criterion for tracheotomy indication and the need for tracheostomy should be reviewed in critical patients [11]. Therefore, the present study aimed to compare the complications, operation times and functional outcomes of PT and ST in elderly patients with respiratory failure.

\section{Materials and Method}

\section{Study design and patients}

The medical records of 47 geriatric patients who underwent tracheostomy at our hospital were retrospectively evaluated in this study. Informed consent was not obtained from patients because of the retrospective study design. The study was approved with a waiver of consent by the Local Ethics Committee (Date: 30.05.2019/ MH 35). Patients below the age of 65 years were excluded from the study. At our hospital, tracheostomies are performed by anesthesia and reanimation specialists and otorhinolaryngologists; ST procedures are performed by the latter. Recently, anesthesia and reanimation specialists have begun performing PT. PT and ST were performed in patients at ICU beds and operating rooms, respectively. No muscle relaxants were used for either procedure.

Patients were sedated with midazolam and propofol, and local anesthesia was applied. Patients who underwent PT and ST were evaluated. Age, sex, indication for ICU admission comorbidity status, APACHE II scores, number of intubated days, intubation time, survival days, operation time, complications during and after the procedure (hemorrhage, subcutaneous emphysema, pneumothorax, hypotension, stoma infection, displacement, mortality, necessity of PT conversion to ST, hypoxia and tracheal stenosis), and the presence of tracheostomy during discharge were recorded. The mean operation time was defined as the time from the skin incision to the insertion of the tracheostomy tube.

\section{Open surgical tracheostomy technique}

The patients were placed in the supine position with their neck extended. The neck is to be fully extended by placing a shoulder roll underneath the upper back area and the head is stabilized on a head ring [12]. Under sterile conditions, the surgical site was covered. The cricoid cartilage and sternal notch were determined, and the midpoint of the line joining these two points was marked.
Local anesthesia was applied to the surgical field. A $1.5 \mathrm{~cm}$ incision was made. Blunt dissection in the midline through the linea alba is carried out and the strap muscles are retracted laterally using Langen beck retractors. The thyroid isthmus comes into view and this can have a variable position and size. It is skeletonized and can be divided either using clamps and transfixion sutures or using monopolar or bipolar diathermy.

The anterior tracheal wall is identified and cleaned. It is useful to identify the cricoid cartilage so to be able to assess the entry point for the tracheotomy. Tracheotomy was usually performed on the $3^{\text {rd }}$ or $4^{\text {th }}$ tracheal rings, and the area was marked by bipolar diathermy. A conservative square tracheal window was created as much as possible. The membrane tissue was carefully cut between the rings. After the tracheal window was opened, secretion was thoroughly aspirated. The assistant was asked to pull the endotracheal tube. A tracheostomy cannula with an appropriate diameter was inserted through the tracheal window. The tracheostomy cuff was then inflated, and bilateral chest enlargement was confirmed by ventilating the lung with the cannula [12].

\section{Percutaneous dilation tracheostomy technique}

The patient is placed in a supine position. To extend the neck, a towel wrapped under the shoulder is placed so that the trachea can be easily palpated, and the cricoid cartilage is better pulled. Tracheal rings are palpated to identify the stern notch and cricoid cartilage. After the patients were positioned, antisepsis of the neck was achieved, and local anesthesia was applied. With the help of a laryngoscope or bronchoscope, the ET cuff was retracted so that it could be seen between the vocal cords. A skin incision of $1-1.5 \mathrm{~cm}$ was made at the level of the second-third tracheal rings. The location of the needle was confirmed by entering the trachea with 2-3mL saline-filled syringe from the midline and aspirating the air. A J-tipped guidewire was advanced through the needle and the needle was removed. A guiding catheter was inserted over the guidewire. All dilatations were performed on this combination of guidewire and guiding catheter. Dilatations were started with a $12 \mathrm{~F}$ dilator and sequential expansion was made up to $36 \mathrm{~F}$ dilator for the $8 \mathrm{~mm}$ tracheostomy cannula. The last tracheostomy cannula with cuff was placed over the combination of guidewire and guiding catheter. The tracheostomy balloon was inflated. Lung was ventilated and bilateral chest enlargement was confirmed [13].

\section{Statistical analyses}

Statistical analyses were performed using the IBM SPSS for Windows 23.0 (USA) software. Categorical data were expressed as numbers and percentages, and continuous data were expressed as mean or ranges. The chi-square test and independent sample t-test were used to compare categorical variables. A p-value of $<0.02$ was considered statistically significant. 


\section{Global Journal of Otolaryngology}

\section{Results}

A total of 47 patients were included in the study. Among them, $23(48.9 \%)$ were male and $24(51.1 \%)$ were female. The mean \pm SD age of the patients was $78.34 \pm 10.233$ (range, 65-98) years. The patients undergoing PT comprised 27 patients [mean \pm SD age $79.78 \pm 10.211$ (range, 65-98) years], and the patients undergoing ST comprised 20 patients [mean \pm SD age $76.40 \pm 10.195$ (range, 65-92) years]. No significant differences were observed between the age, sex, APACHE II score and other demographic characteristics of the patients in the patients undergoing PT and ST (Table 1). The diagnoses of the patients during hospitalization were respiratory (48.9\%), neurological $(42.6 \%)$, and cardiac diagnoses $(6.4 \%)$, respectively.

Table 1: Demographic characteristics of patients in the patients undergoing ST and PT.

\begin{tabular}{|c|c|c|c|c|}
\hline & Total $(\mathbf{n}=\mathbf{4 7})$ & PT (n= 27) & ST (n= 20) & p value \\
\hline Age (years), mean \pm SD & $78.34 \pm 10.23$ & $79.78 \pm 10.21$ & $76.40 \pm 10.19$ & 0.268 \\
\hline Sex (male/female), n & $23 / 24$ & $17-$-Oct & $13 / 7$ & 0.054 \\
\hline APACHE II score, mean \pm SD & $31.08 \pm 10.46$ & $30.70 \pm 10.34$ & $31.60 \pm 10.87$ & 0.775 \\
\hline Tracheostomy opening duration (min), mean \pm SD & $16.39 \pm 6.09$ & $11.2 \pm 2.8$ & $23.4 \pm 3.2$ & 0.001 \\
\hline Duration from intubation to tracheostomy (days), mean \pm SD & $20.17 \pm 9.80$ & $20.81 \pm 10.96$ & $19.35 \pm 8.26$ & 0.623 \\
\hline Duration from tracheostomy to death (days), median (range) & $46(1-351)$ & $50(1-351)$ & $40(9-135)$ & 0.662 \\
\hline Duration of stay in the intensive care unit (days), mean \pm SD & $63.21 \pm 54.95$ & $66.89 \pm 66.35$ & $58.25 \pm 35.23$ & 0.6 \\
\hline Mortality rate (\%) & $74.50 \%$ & $77.80 \%$ & $70.00 \%$ & 0.477 \\
\hline
\end{tabular}

PT: percutaneous tracheostomy; ST: surgical tracheostomy.

Most patients had comorbidity (85.1\%). The most common comorbidities were malignancy (38.3\%) and heart failure (25.5\%). No significant difference was observed between PT and ST patients in terms of primary diagnosis and comorbidity frequency ( $p>0.05)$. Overall, tracheostomy was performed at $20.85 \pm 9.507$ days (mean \pm SD) after intubation. The median duration from tracheostomy to death was 46 (range, 1-351) days and from tracheostomy to discharge was 33 (range, 17-81) days; the corresponding median values were 50 (range, 1-351) and 32 (range, 27-37) days, respectively, in the patients undergoing PT and 40 (range, 9-135) and 34 (range, 17-81) days, respectively, in the patients undergoing ST. No significant differences were observed for this parameter between the two groups ( $p>0.05)$. The proportion of patients who died within the first 30 days of tracheostomy was $55 \%$. Overall, $74.5 \%(n=35)$ of the patients died before discharge from the ICU, including $87.78 \%(\mathrm{n}=21)$ in the patients undergoing PT and $70 \%(n=14)$ in the patients undergoing ST (Figure 1).

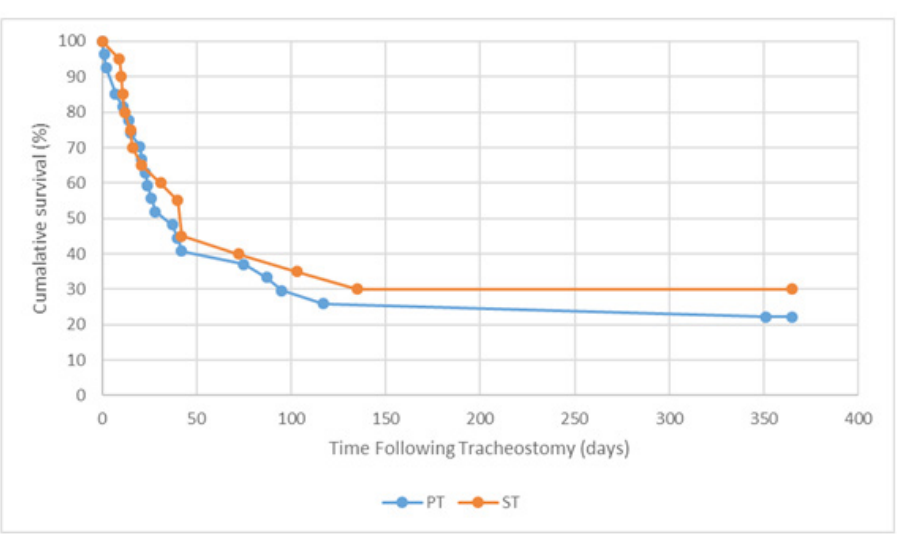

Figure 1: Survival times during intensive care hospitalization among patients undergoing percutaneous and surgical tracheostomy. PT: percutaneous tracheostomy; ST: surgical tracheostomy.

After tracheostomy, 22.22\% $(n=6)$ of the patients in the patients undergoing PT and $25 \%(n=5)$ of the patients in the patients undergoing ST remained attached to the ventilator and were discharged as tracheostomized. No significant differences were noted in terms of discharge time and mortality rate between the groups $(\mathrm{p}>0.05)$. Only one patient undergoing ST was decannulated and discharged with continuous vocal function. No patients were decannulated during ICU follow-up. Survival status 
was not followed up after discharge. The mean \pm SD operation times were $23.4 \pm 3.2 \mathrm{~min}$ for ST and $11.2 \pm 2.8 \mathrm{~min}$ for PT; these differences were statistically significant $(\mathrm{p}<.001)$.

No serious hypoxia (defined as oxygen saturation values of $<90 \%$ ) was observed in the groups. Intraoperative complications associated with tracheostomy were more frequent in the patients undergoing PT (22.22\%) than in the patients undergoing
ST (15.00\%). Conversely, postoperative complications were more frequent in the patients undergoing ST $(25.00 \%)$ than in the patients undergoing PT (11.11\%; Table 2). However, the differences in intraoperative and postoperative complications between the groups were not significant ( $p>0.05)$. PT was converted to ST in 1 patient because the tracheostomy tube was inserted incorrectly.

Table 2: Intraoperative and postoperative complications in the patients undergoing ST and PT.

\begin{tabular}{|c|c|c|c|c|}
\hline Intraoperative Complications & Total $(n=47)$ & PT $(n=27)$ & ST $(n=20)$ & p value \\
\hline Hypoxemia & $8.51 \%$ & $7.41 \%$ & $10.00 \%$ & \multirow{6}{*}{0.787} \\
\hline Bleeding & $4.26 \%$ & $3.70 \%$ & $5.00 \%$ & \\
\hline Death & $2.13 \%$ & $3.70 \%$ & $0.00 \%$ & \\
\hline Placement in the wrong position & $2.13 \%$ & $3.70 \%$ & $0.00 \%$ & \\
\hline Conversion from PT to ST & $2.13 \%$ & $3.70 \%$ & $0.00 \%$ & \\
\hline Total & $19.15 \%$ & $22.22 \%$ & $15.00 \%$ & \\
\hline Postoperative Complications & Total $(n=47)$ & PT (n = 27) & ST $(n=20)$ & p value \\
\hline Subcutaneous emphysema & $4.26 \%$ & $0.00 \%$ & $10.00 \%$ & \multirow{5}{*}{0.272} \\
\hline Tracheal stenosis & $2.13 \%$ & $0.00 \%$ & $5.00 \%$ & \\
\hline Tracheal oesophageal fistula & $2.13 \%$ & $3.70 \%$ & $0.00 \%$ & \\
\hline Minor bleeding & $8.51 \%$ & $7.41 \%$ & $10.00 \%$ & \\
\hline Total & $17.02 \%$ & $11.11 \%$ & $25.00 \%$ & \\
\hline
\end{tabular}

PT: percutaneous tracheostomy; ST: surgical tracheostomy.

\section{Discussion}

In this study, PT and ST techniques were compared in terms of complications in elderly patients who underwent tracheostomy. Postoperative complications were higher in patients undergoing ST, and intraoperative complications were higher in patients undergoing PT. However, this difference was not statistically significant. A previous study has demonstrated that operation times and complications are reduced with PT compared with ST [14]. There is no consensus regarding the optimal tracheostomy method for minimizing complications [1]. There are special cases wherein ST remains the first choice; this method should be preferred for pediatric patients and patients who have coagulation abnormalities (better hemostasis control), require high levels of respiratory support (fraction of inspired oxygen $<70 \%$ and positive end-expiratory pressure $<10 \mathrm{~cm} \mathrm{H}_{2} \mathrm{O}$ ), have cervical spinal injury (difficulty in positioning) or are obese (body mass index $>30 \mathrm{~kg} / \mathrm{m}^{2}$ ] [15].

In some studies, it was observed that PT technique was applied under the guidance of fiberoptic bronchoscopy. Besides the advantages of fiberoptic bronchoscopy, especially in obese patients, increasing the success of the procedure and reducing the risk of complications such as pneumothorax, posterior wall damage, disadvantages such as increased costs, prolonged procedure time, hypoventilation, hypercarbia and respiratory acidosis [16,17]. In our study, we did not use fiberoptic bronchoscopy as a guide during PT technique. In a meta-analysis of 48 studies, PT was associated with more intraoperative complications and fewer postoperative complications than ST, and the most common intraoperative complications during PT were misplacement and hemorrhage [18]. In a study of 132 patients, the complication rate of PT was $12.9 \%$. Hypoxemia and bleeding were the most common complications during the procedure, whereas subcutaneous emphysema and minor bleeding were the most common complications after the procedure [19]. In another study, postoperative complications were less frequent with PT than with ST [20]. Studies have reported that the incidence of mortality associated with PT ranges from $0 \%-8 \%[15,21]$.

In our study, a patient died within the first 24 hours. This patient was an 89-year-old patient who was followed up for COPD, who did not receive inotropic support on the 42nd day of intensive care. Bleeding and hypoxemia were more common in patients undergoing ST. A tracheoesophageal fistula developed postoperatively in 1 patient who underwent PT. Among patients undergoing ST, one patient developed tracheal stenosis and two developed subcutaneous emphysema. Minor bleeding and total postoperative complications associated with tracheostomy were more common in patients undergoing ST than in those undergoing PT. 
Among these complications, tracheal stenosis and tracheal oesophageal fistula are among the late complications of tracheostomy, while other complications are complications seen during the procedure. Since the average duration of stay in the intensive care unit of the patients included in the study was long (mean \pm SD: $63.21 \pm 54.95$ days), these 2 complications developed in 2 different patients during ICU follow-ups. In the literature, cases with stenosis developed on the $40^{\text {th }}$ day after the procedure have been reported [22]. In one of our patients, tracheal oesophageal fistula developed on the $37^{\text {th }}$ day of the procedure, and on the $42^{\text {nd }}$ day of the procedure, tracheal stenosis developed in the other patient.

Several previous studies have compared the operation times of PT and ST. In one study, the operation times were 22 and 17 min for ST and PT, respectively [23]. In another study, the mean \pm SD operation times were reported to be $21.2 \pm 2.9$ and $12.4 \pm 3.2 \mathrm{~min}$ for ST and PT, respectively [24]. One factor affecting operation times is the expertise of surgeons. In the present study, all the ST procedures were performed by the same experienced otorhinolaryngologist, whereas all the PT procedures were performed by the same experienced anesthesia and reanimation specialist. The mean \pm SD operation times were $23.4 \pm 3.2$ and 11.2 $\pm 2.8 \mathrm{~min}$ for ST and PT, respectively. In terms of the operation time, a statistically significant difference was detected between the two methods. With shorter operating times, PT is an alternative for ST.

In the present study, only 1 patient underwent decannulation; therefore, the decannulation rate could not be evaluated. We attributed the low decannulation rate to the fact that the included sample comprised of geriatric patients. There are studies showing that most of the expectations from tracheostomy (ventilation weaning, decannulation and quality of life) are not met in critical patients [25]. For this reason, decannulation is often not practiced. In the literature, there is no publication showing a significant relationship between decannulation time and tracheostomy technique.

Examination of published studies and current guidelines indicates that elective tracheostomy is most performed because of intubation lasting for more than seven days [11-14,26]. There is no clear consensus on the timing of tracheostomy (early or late), which has been reported to have no effect on mortality [26-28]. A study of 78 elderly patients (mean \pm SD age $77.62 \pm 11.5$ years) with severe and chronic comorbidities who underwent tracheostomy reported a mean \pm SD timing of $16.7 \pm 9.0$ days of intubation [11] In the present study, tracheostomy was performed at mean \pm SD $20.17 \pm 9.80$ days after intubation, which is consistent with that reported in the existing literature.

Elderly patients frequently have multiple comorbidities and high rates of malignancy, which lead to high mortality rates [29]. In one study, an average of $30 \%$ of elderly patients who were followed up in the intensive care unit and discharged, even if they got out of the intensive care unit, died within the first three months due to their underlying chronic diseases [30]. It was observed that $55 \%$ of the patients included in our study died within the first 30 days after tracheostomy. We think that this situation is due to the presence of comorbidity, especially malignancy and heart failure, in most patients (85.1\%) during their admission to ICU. There was no difference between the two patient groups in terms of comorbidities.

A previous study on geriatric patients reported that the median time from tracheostomy to death was 31 (range, 2-256) days and from tracheostomy to hospital discharge was 62 (range, 8-235) days [11]; the corresponding median time were 46 (range, 1-351) and 33 (range, 17-81) days, respectively, in our study. The time from tracheostomy to hospital discharge noted in the present study is consistent with that reported in the literature; however, the time from tracheostomy to death is much higher. The longer time to discharge was attributed to the inability of patients to be admitted to palliative care units. Palliative care patients stay longer and often die in ICUs because palliative care units and home care services in Turkey are insufficient [31].

A previous study has reported that the APACHE II score was a good indicator of the need for tracheostomy during the first few days of ventilation, suggesting that early tracheostomy should be provided to patients presenting with shock and APACHE II scores $>25$ [32]. In the present study, the mean \pm SD APACHE II score was $31.08 \pm 10.46$. The present study has some limitations. The survival and decannulation status of patients discharged as tracheostomized were not followed up.

\section{Conclusion}

This study found that operation time with PT was shorter than with ST. No significant difference was observed between the complications of these two procedures. However, it is an advantage that PT can be applied to the patient bed in intensive care units. In the case of long-term intubation among elderly patients, planned tracheostomy should be performed, considering clinical benefits and risks, knowing that expectations from tracheostomy may not be met most of the time..

\section{References}

1. Ramakrishnan N, Singh JK, Gupta SK, Bhalla V, Singh D, et al. (2019) Tracheostomy: Open Surgical or Percutaneous? An Effort to Solve the Continued Dilemma. Indian Journal of Otolaryngology and Head \& Neck Surgery 1-7.

2. Klotz R, Probst P, Deininger M, Klaiber U, Grummich K, et al. (2018) Percutaneous versus surgical strategy for tracheostomy: a systematic review and meta-analysis of perioperative and postoperative complications. Langenbecks Arch Surg 403(2): 137-149.

3. Kaya C, Köksal E, Üstün F (2014) Tracheostomy in the practice of anesthesia and intensive care. Journal of Harran University Medical Faculty 11: 172-183.

4. Alabi B, Afolabi O, Dunmade A, Omokanye H, Ajayi I, et al. (2018) Indications and outcome of tracheostomy in Ilorin, North Central Nigeria: 10 years review. Ann Afr Med 17(1): 1-6. 
5. Massick DD, Yao S, Powell DM, Griesen D, Hobgood T, etal.(2001) Bedside tracheostomy in the intensive care unit: a prospective randomized trial comparing open surgical tracheostomy with endoscopically guided percutaneous dilational tracheotomy. Laryngoscope 111(3): 494-500.

6. Epstein SK (2005) Late complications of tracheostomy. Respiratory care 50(4): 542-549.

7. Oliver ER, Gist A, Gillespie MB (2007) Percutaneous versus surgical tracheotomy: an updated meta-analysis. Laryngoscope 117(9): 15701575 .

8. Durbin CG Jr (2005) Early complications of tracheostomy. Respir care 50(4): 511.

9. Cistir AA, Akgün N, Özkaraman M, Demirel M, Karadoğan F, et al. Geriatric Patients and Problems in Weaning from Mechanical Ventilation. BMJ 2(1): 14-20.

10. Doğan S, Can H, Doğan N, Gönüllü M (2013) Comparison of the Mortality Rates for Patients Aged 65 Years and Over and Aged Below 65 Years, Hospitalized in the Intensive Care Unit. Turkey Clinical J Med Sci 33(6): 1408-1413.

11. Baskin JZ, Panagopoulos G, Parks C, Rothstein S, Komisar A (2004) Clinical outcomes for the elderly patient receiving a tracheotomy. Head Neck 26(1): 71-76.

12. Muscat K, Bille A, Simo R (2017) A guide to open surgical tracheostomy. Open Thoracic Surgery 1: 112.

13. Walz M, Peitgen K, Thürauf N, Trost H, Wolfhard U, et al. (1998) Percutaneous dilatational tracheostomy-early results and long-term outcome of 326 critically ill patients. Intensive Care med 24(7): 685690.

14. Franćois B, Clavel M, Desachy A, Puyraud S, Roustan J, et al. (2003) Complications of tracheostomy performed in the ICU: subthyroid tracheostomy vs surgical cricothyroidotomy. Chest 123(1): 151-158.

15. Durbin CG (2005) Techniques for performing tracheostomy. Respiratory care 50(4): 488-496.

16. Öksüz, Hafize, et al. (2010) Fiberoptic Bronchoscope versus Lightwand Guided Techniques for Percutaneous Dilational Tracheostomy. Journal of the Turkish Anaesthesiology \& Intensive Care Society-JTAICS 38: 2.

17. Paran H, Butnaru ci, Hass I, Afanayv A, Gutman M (2004) Evaluation ol a modified percutaneous tracheostomy technique without bronchoscopic guidance. Chest 26(3): 868-871.

18. Dulguerov P, Gysin C, Perneger TV, Chevrolet J C (1999) Percutaneous or surgical tracheostomy: a meta-analysis. Critical care medicine 27(8): 1617-1625.
19. Karasu D, Yılmaz C, Baytar C, Korfalı G (2018) Retrospective Analysis of Percutaneous Tracheostomi Cases in Intensive Care Unit. Turk J Intensive Care 16(3): 83-87.

20. Freeman BD, Isabella K, Lin N, Buchman TG (2000) A meta-analysis of prospective trials comparing percutaneous and surgical tracheostomy in critically ill patients. Chest 118(5): 1412-1418.

21. Richard S (2005) Interventions and Techniques in Intensive Care. ( $3^{\text {rd }}$ ) Edition, Nobel Medical Bookstore pp 150-159.

22. Kırca, Hülya, et al. (2018) Tracheotomy in the Intensive Care Unit: Indications, Complications and Prognosis. J Turk Soc Intensive Care 16: 17-25.

23. Antonelli M, Michetti V, Di Palma A, Conti G, Pennisi MA, et al. (2005) Percutaneous translaryngeal versus surgical tracheostomy: a randomized trial with 1-yr double-blind follow-up. Critical care med 33(5): 1015-1020.

24. Hacı C, Açıkalın RM, Akkoç İ, Toptaş M (2018) Investigation of Advantages and Disadvantages of Percutaneous and Surgical Tracheostomies. Haseki Tip Bulteni 56(1): 65.

25. Mitchell RB, Hussey HM, Setzen G, Jacobs IN, Nussenbaum B, et al. (2013) Clinical consensus statement: tracheostomy care. Otolaryngol Head Neck Surg 148(1): 6-20.

26. Alberti PW (1977) Hearing aids and aural rehabilitation in a geriatric population. J Otolaryngol Suppl 4: I.

27. Clec h C, Alberti C, Vincent F, Garrouste Orgeas M, De Lassence A, et al. (2007) Tracheostomy does not improve the outcome of patients requiring prolonged mechanical ventilation: a propensity analysis. Crit care med 35(1): 132-138.

28. Griffiths J, Barber VS, Morgan L, Young JD (2005) Systematic review and meta-analysis of studies of the timing of tracheostomy in adult patients undergoing artificial ventilation. BMJ 330(7502): 1243.

29. Parks R, Rostoft S, Ommundsen N, Cheung K L (2015) Peri-operative management of older adults with cancer-the roles of the surgeon and geriatrician. Cancers 7(3):1605-1621.

30. Somme D, Maillet JM, Gisselbrecht M, Novara A, Ract C, et al. (2003) Critically ill old and the oldest-old patients in intensive care: short-and long-term outcomes. Intensive Care Med 29(12): 2137-2143.

31. Kepekci AB, Erdoğan E, Zıvalı M (2019) Frequency of palliative care patients in a second level intensive care unit: Retrospective study. JARSS 27(3): 193-197.

32. Heffner JE (2003) Tracheotomy application and timing. Clinin chest Med 24(3): 389-398.

\section{Your next submission with Juniper Publishers will reach you the below assets}

- Quality Editorial service

- Swift Peer Review

- Reprints availability

- E-prints Service

- Manuscript Podcast for convenient understanding

- Global attainment for your research

- Manuscript accessibility in different formats

( Pdf, E-pub, Full Text, Audio)

- Unceasing customer service

Track the below URL for one-step submission https://juniperpublishers.com/online-submission.php 